\title{
The lack of value of hepato-jugular reflux as a sign of heart failure
}

\author{
S. P. SINGH \\ M.B., F.R.C.P. \\ R. HAIDER* \\ M.R.C.P.
}

United Birmingham Hospitals and Birmingham Regional Hospital Board; Division of Medicine, University of Birmingham

\begin{abstract}
Summary
An investigation was carried out of the diagnostic value of hepato-jugular reflux in patients with heart failure.

The effect of abdominal compression on superior vena cava pressure was assessed in patients with and without heart failure. In four of the six patients without congestive cardiac failure, during compression, whether applied in the right hypochondrium or left iliac fossa, there was a significant rise in vena cava pressure. A similar response was also observed in three patients with congestive heart failure.

Hepato-jugular reflex is not a reliable sign of incipient heart failure.
\end{abstract}

RONDOT (1898) described the diagnostic value of hepatic compression in patients with heart failure. Pasteur (1885) had previously described elevation of pressure in the neck veins in patients with tricuspid incompetence. The sign of filling of the jugular veins when pressure is applied to the abdomen in the region of the right hypochondrium was called hepato-jugular reflux. Since Rondot's description, this sign has been used in clinical practice to confirm the presence or absence of congestive heart failure in borderline cases, when peripheral oedema is absent and there is no visible increase in the jugular venous pressure. Few scientific data are available to confirm the accuracy of this sign. The present investigations describe the effect of abdominal compression on the central venous pressure in normal subjects and patients with heart failure.

\section{Material and methods}

During routine cardiac catheterization or coronary angiocardiography in eleven patients, the abdomen was first compressed with the palm of the hand at the lower right subcostal margin and later in the left iliac fossa, from 15 to $100 \mathrm{sec}$, and the pressure in the superior vena cava was measured. In two patients with heart failure and two normal patients, additional measurements were made from the right ventricle, pulmonary artery, wedge, left venticle and the ascending aorta.

\footnotetext{
* Present address: Department of Clinical Cardiology, Royal Postgraduate Medical School, Hammersmith Hospital, London.
}

These measurements were made with the patient lying flat or reclining at 30 degrees. The zero reference point was taken at the mid-axillary line in the fourth intercostal space. Six children and young patients had a general anaesthetic. The remaining five were sedated with intravenous valium and were instructed to breathe freely and not to resist when the pressure was applied on the abdomen. In six patients the cardiac lesion was congenital and in two rheumatic; three had ischaemic heart disease.

Patients were divided into two categories. The $O$ first group of six patients had no evidence of heart failure and end-diastolic pressures in the right ventricle and the mean central venous pressures were normal. Similarly, the mean pulmonary wedge pressure (indirect left ventricular diastolic pressure) was not elevated (Group I).

In the second group of five patients, three had physical signs of congestive cardiac failure an $\$$ raised central venous pressure (Group IIA). In two patients, there was clinical evidence of early left ventricular failure and raised pulmonary wedge pressure (Group IIB).

\section{Results}

Group I (six patients without congestive cardiac failure): The mean central venous pressure was $2-5 \mathrm{mmHg}$. During compression in the right hypochondrium or left iliac fossa, there was a steep rise in both free and mean pressures $(3-5 \mathrm{mmHg}$ ) in four patients. After the initial steep rise (Fig. 1), there was a slight fall, and the level dropped to control levels after release of compression. Two patients in this group had additional measurements - there was an increase in the right ventricular enddiastolic pressure (Fig. 2), free and mean pulmonary artery and wedge pressures and left atrial pressure (Fig. 3). There was a slight and probably not significant rise in the systemic arterial pressure. During compression in four patients, distension and engorgement of the external jugular veins was noted.

Group IIA (three patients with congestive heart failure): The mean central venous pressure varied from 8 to $12 \mathrm{mmHg}$ and rose by an average mean of $5 \cdot 5(4-7 \mathrm{mmHg})$. The right ventricular, pulmonary artery and wedge pressure responses were similar to that noted in normal patients (Group I). 
Superior veno covo
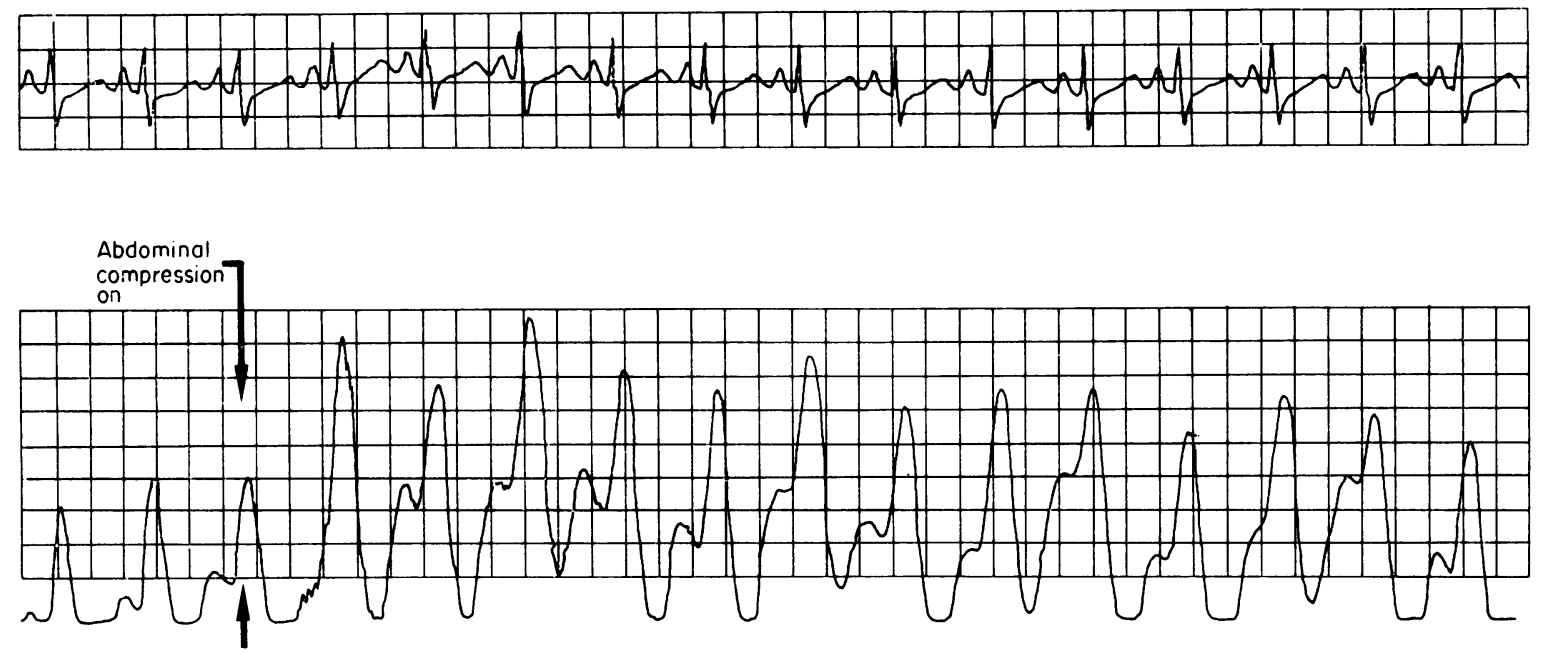

FIG. 1. The effect of compression in the right hypochondrium in a patient without heart failure. After a steep rise there was some fall; the pressure returned to normal levels after the release of pressure (ordinate: CVP, scale as Fig. 2).
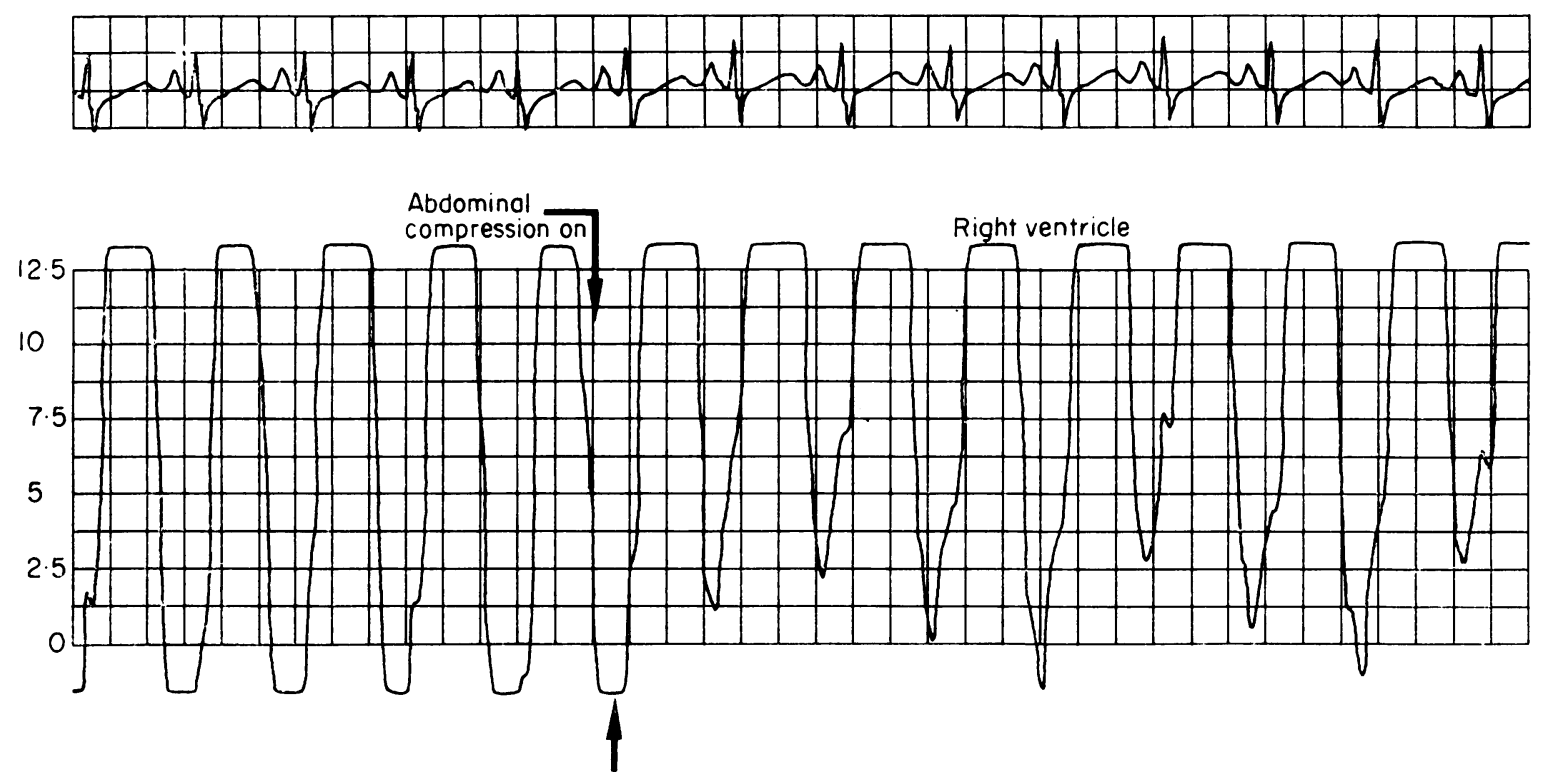

FIG. 2. The effect of compression on the RV end diastolic pressure: a rise of approx $4 \mathrm{mmHg}$ (patient without heart failure).

Group IIB: In these two patients with elevated pulmonary (left atrial) pressure, the central venous pressure was normal. On abdominal compression in the right hypochondrium and the left iliac fossa, engorgement of neck veins with simultaneous rise in central venous pressure was noted.

\section{Discussion}

The present study shows that there is elevation of central venous pressure on abdominal compression, both in the absence and in the presence of cardiac failure. The response was similar whether the right hypochondrium or left iliac fossa was compressed. It is interesting that Wood (1956) had previously confirmed that pressure anywhere over the abdomen and not only over the liver region will increase the jugular venous pressure. Landis \& Hortenstine (1950) found that pressure on any part 


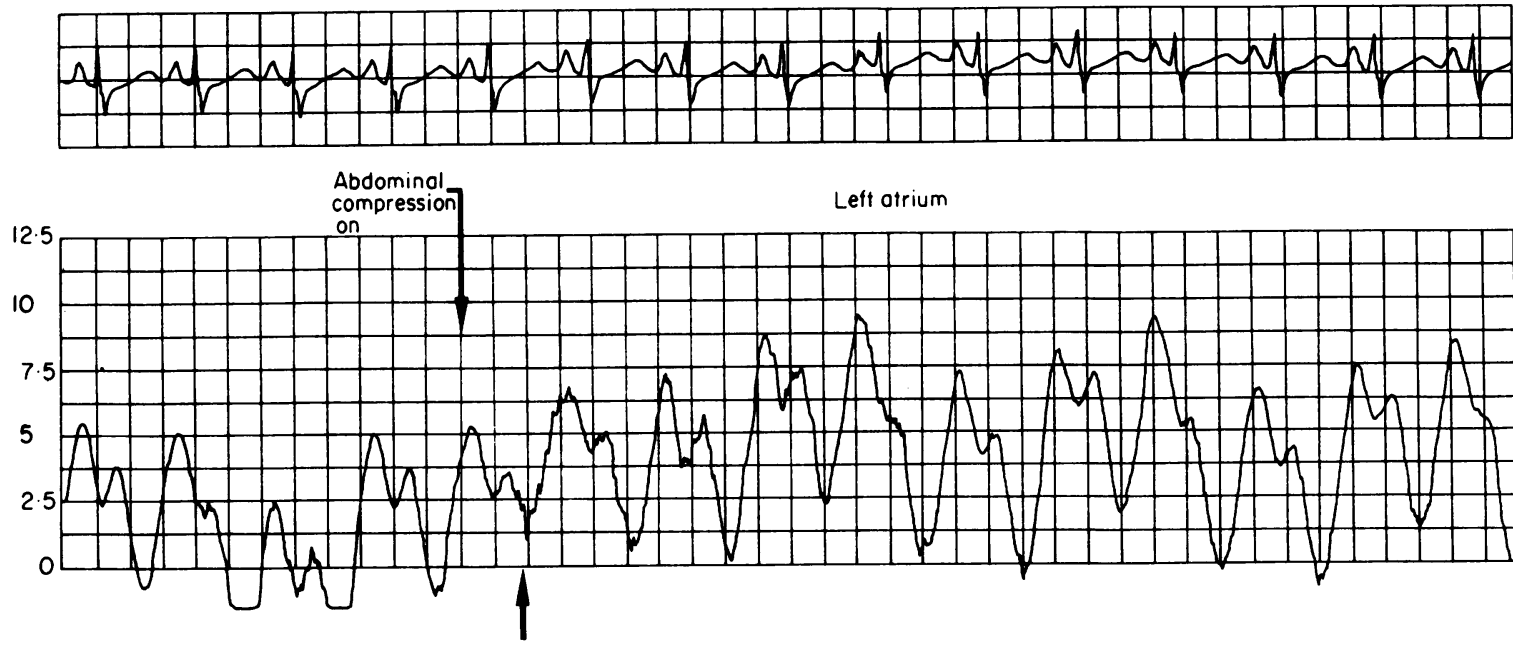

FIG. 3. Left atrial pressure recording, showing a rise on abdominal compression (patient without heart failure).

of the abdomen is an ideal method of increasing venous return.

The increased central venous pressure could result from an increased volume of blood being released by direct squeezing of the abdominal veins, increased venous tone or by a reflex Valsalva effect.

The latter can occur during breath-holding, when sudden pressure is applied to the abdominal muscles, and this increases the intrathoracic pressure. In this study, the Valsalva effect was eliminated as the majority of our patients had a general anaesthetic and the rest were well sedated and instructed to continue to breathe normally. In the recumbent position, the lower limbs contain $300-800 \mathrm{ml}$ of blood, which is added to the effective circulation in the supine position. Our previous study of patients with acute myocardial infarction showed that, in two patients who had no evidence of cardiac failure and with normal right atrial and wedge pressures, a change from the recumbent to the supine position resulted in minor increases of 1 and $2 \mathrm{mmHg}$ respectively (Eddy \& Singh, 1969). It would seem that the 'squeezing effect' on the abdominal veins must add a much larger quantity to the venous return to the heart than positional change to produce the pressure effect seen in our four normal subjects. It is difficult to know the exact amount of blood that was added to the circulation during compression, as we did not measure the cardiac output.

The rise in pulmonary artery and wedge pressures occurred in both groups but was most marked in patients with cardiac failure. In the latter group, the mechanism is perhaps similar to that seen with change of position; the additional volume of blood added to the circulation was sufficient to further increase the pulmonary artery and wedge pressures.
In normal subjects, besides volume load, other factors-such as increased venoconstriction-might have caused these changes.

Burch \& Ray (1951) suggested that not only the high venous pressure, but also the rise due to abdominal compression in patients with failure was due in part to increased venous tone. It is possible that a similar mechanism may have played a part if elevating central venous pressure.

Most of the previous studies have suggested that hepato-jugular reflux is present only in patients with cardiac failure (Hitzig, 1945; Hultgren, 1950; Burch \& Ray, 1954). Burch \& Ray (1954) found that a normal person will experience only slight, if any, elevation, and in many instances his venous pressure will decline. In these studies, however, only the antecubital venous pressure was measured, which is often different from the central venous pressure (Pedrsen, 1952). Also saline manometric observations are not as accurate as the modern method of measuring and recording intravascular pressures. In previous studies, the patients were not anaesthetized but were instructed to breathe with their mouths open. It is possible that the fall in pressure in some of these patients could have been due to increased respiratory effort giving rise to decreased intrathoracic pressure. Hitzig (1945), in some normal patients, observed some rise in the venous pressure which did not persist for more than $45 \mathrm{sec}$. We applied the compression for a longer period of up to $100 \mathrm{sec}$ in one normal patient, but did not notice any appreciable fall (Fig. 4). Mathews \& Hampson (1958) observed the effect on the central venous pressure by placing a lead weight on the abdomen in normal patients and cases of congestive cardiac failure. They found the effect on the venous pressure (5) 


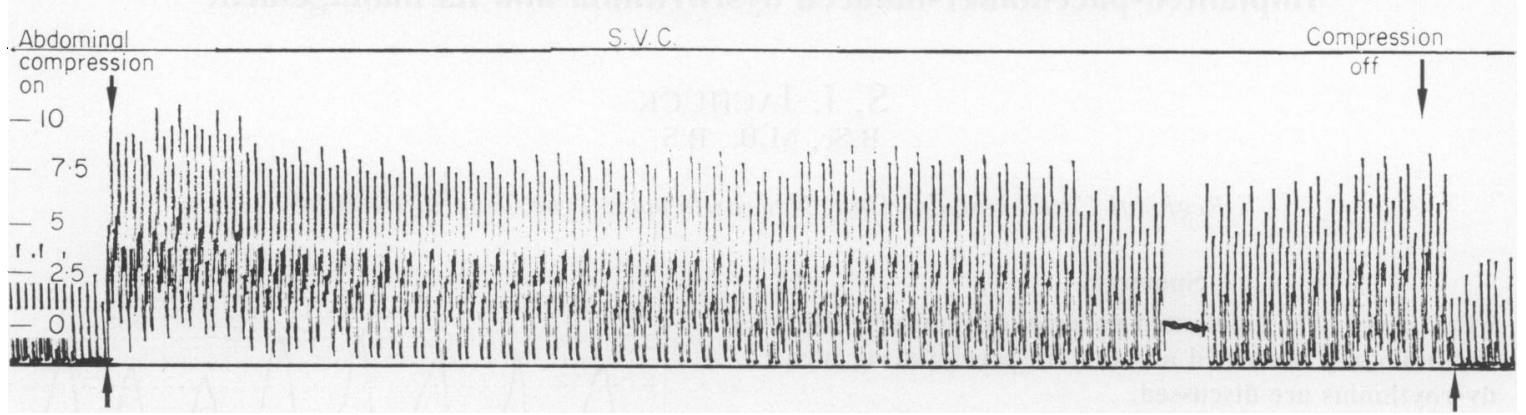

FIG. 4. Prolonged abdominal compression lasting $100 \mathrm{sec}$ in a patient without heart failure shows sustained rise in the central venous pressure (paper speed-2.5 mm/sec). S.V.C., superior vena cava.

was determined by the simultaneous change in thoracic pressure and, as the latter was unpredictable even in the same subjects, observations of jugular venous pressure during hepatic compression are unreliable as an early sign of heart failure. They also observed that, in an athletic medical student, the central venous pressure rose by $9 \mathrm{~cm}$ when a weight was placed on his abdomen, and $22 \mathrm{sec}$ elapsed before it returned to its resting level. Wahi, Bhargava \& Mahindra (1969) also recorded an increase in the right ventricular end-diastolic pressure (the ' $a$ ' wave in right atrial pressure tracings) in some patients who were not in cardiac failure.

This investigation suggests the difference between the effects of compression on venous pressure between normal subjects and those with congestive heart failure may be quantitative rather than qualitative, like that of exercise, and their significance cannot always be assessed accurately by mere clinical observation of the neck veins (Landis \& Hortenstine, 1950). Our studies also confirmed that, in congestive cardiac failure, the rise in venous pressure was more marked (average mean of 5.5 $\mathrm{mmHg}$ ) than in four of the six normal subjects (4 mmHg). Lewis (1946) noticed that, in many normal subjects, if they lean forward as they sit, prominent pulsations appear in the jugular veins because pressure on the abdomen in this posture displaces blood into the neck.

Wood (1956) also pointed out that tight corsets, abdominal binders, tight trousers, ascites, pregnancy or even gross flatulence may all produce this effect. To see alleged 'heart failure' immediately relieved by undoing the top trouser buttons can be both embarrassing and instructive. We conclude that hepato-jugular reflux is not a reliable sign of early or incipient heart failure, but it is of course of value for distinguishing venous from arterial pulsation in doubtful cases.

\section{References}

BURCH, G.E. \& RAY, C.T. (1951) A consideration of mechanism of congestive heart failure. American Heart Journal, 41, 918.

BuRCH, G.E. \& RAY, C.T. (1954) Mechanism of the hepatojugular reflux test in congestive heart failure. American Heart Journal, 48, 373.

EDDY, J.D. \& SiNGH, S.P. (1969) Nursing posture after acute myocardial infarction. Lancet, ii, 1378.

Hitzig, W.M. (1945) Venous pressure curves in normal and abnormal circulatory states. Normal venous pressure curves and negative 'Hepato-jugular reflux phenomenon'. Journal of the Mount Sinai Hospital, 12, 309.

Hultgren, H.N. (1950) Effect of increased venous return. American Heart Journal, 39, 592.

LANDis, E.M. \& HoRTenstine, J. C. (1950) Functional significance of venous pressure. Physiological Reviews, 30, 1 .

LEwIS, T. (1946) Disease of the Heart, p. 14. Macmillan, London.

Lian, C. \& Blondel, A. (1925) Presse médicale, 33, 481.

Mathews, M.B. \& Hampson, J. (1958) Hepatojugular reflux. Lancet, $\mathbf{i}, 873$.

Pasteur, W. (1885) Note on a new physical sign of tricuspid regurgitation. Lancet, ii, 524.

Pedrsen, A. (1952) The pressure in the central veins and its bearing on peripheral venous pressure measurement. Acta medica scandinavica, Suppl. 266, 829.

RoNDOT, E. (1898) Le reflux hepato-jugulaire. Gazette hebdomadaire des sciences médicales de Bordeaux, 19, 567.

Wahi, P.L., Bhargava, K.C. \& Mahindra, S. (1969) Cardiac changes in progressive muscular dystrophy. A hemodynamic study. Israel Journal of Medical Sciences, 5, 797.

Wood, P. (1956) Disease of the Heart and Circulation, pp. 44-46. Eyre and Spottiswood, London. 